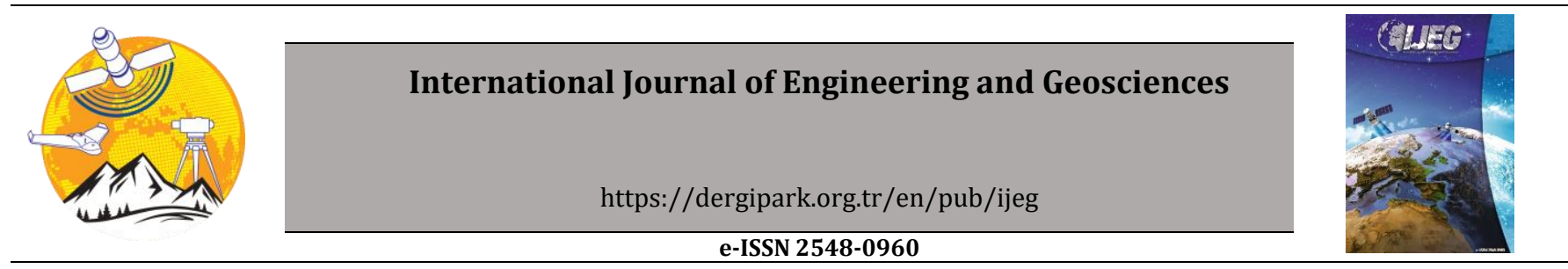

\title{
Fully optimized multilayer radar absorber design using multi-objective abc algorithm
}

\author{
Enes Yigit ${ }^{1}$, Huseyin Duysak ${ }^{* 1}$ \\ ${ }^{1}$ Karamanoglu Mehmetbey University, Engineering Faculty, Department of Electrical-Electronics Engineering, Karaman, Turkey
}

\section{Keywords}

Multi-objective optimization Pareto front

Radar absorbing material

(RAM)

Triple objective $\mathrm{ABC}$

\begin{abstract}
Main purpose of the design of multi-layer radar absorber (MRA) by means of metaheuristic optimization algorithms is to minimize both the total thickness (TT) of MRA and the maximum reflection coefficients for transverse electric (RTE) \& transverse magnetic (RTM) polarizations at any oblique angle of incidence. For this purpose, sequence and thicknesses of layers of the MRA have been optimized by either single-objective approach based on combining all objectives or double-objective approach in which TT is evaluated separately from the reflection coefficients. In this study, triple-objective artificial bee colony (TO-ABC) algorithm integrated with Pareto front technique is proposed for fully optimized MRA design. Thus, both RTE, RTM and TT are simultaneously minimized by optimizing thickness, sequence and number of the layers. To demonstrate the superiority of TO-ABC, 3 types of MRAs operating at the frequency ranges of $2-18 \mathrm{GHz}$ for each angle of incidence from $0^{0}$ to $60^{\circ}$ are optimized and compared with the literature. Furthermore, 4 different real MRAs are also optimized using real materials given in the literature. Thanks to the developed graphical user interface and TO-ABC algorithm, despite the limited number of materials, all possible solutions providing the specified parameters are easily achieved and successful MRA structures are designed.
\end{abstract}

\section{INTRODUCTION}

Electromagnetic (EM) stealth technology is an important issue which has increased its popularity in the last quarter century and has many commercial and military applications. The main purpose of this technology is to reduce the EM energy scattered from the target as much as possible and to make the target invisible to the radar receiver. Within this scope, multilayer radar absorbers (MRA) have become very popular in recent years due to their superior absorption capabilities (Yigit and Duysak, 2019a). To produce the MRA having low thickness and low reflection properties, the sequence and thickness of the materials used in the layers should be optimally determined. For this purpose, natural-inspired optimization algorithms such as artificial bee colony (ABC)(Toktas et al., 2018), central force algorithm (CFO) (Asi and Dib, 2010), particle swarm optimization (PSO) (Goudos and Sahalos, 2006a), genetic algorithm (GA) (Kern and Werner, 2003) and differential evolution (DE) (Goudos, 2009) and also surrogate-based optimization(Toktas et al., 2019) have been successfully applied to various MRA designs operating at different frequency ranges between 0.2-18 GHz (Asi and Dib, 2010; Goudos, 2009; Goudos and Sahalos, 2006a, 2006b; Jiang et al., 2009; Kern and Werner, 2003; Michielssen et al., 1993; Ranjan et al., 2018; Roy et al., 2016, 2015; Toktas et al., 2019, 2018; Weile et al., 1996; Yigit and Duysak, 2019a). Most of these studies were performed either for normal incidence or for a limited angle of incidence. Broad-band and wideangle MRAs should be designed for practical implementations of the stealth technologies. However, the design of broad-band and wide-angle MRA with as thin as possible is a challenging problem. In all of the studies given in references (Asi and Dib, 2010; Goudos, 2009; Goudos and Sahalos, 2006a, 2006b; Jiang et al., 2009; Kern and Werner, 2003; Michielssen et al., 1993; Ranjan et al., 2018; Roy et al., 2016, 2015; Toktas et al., 2019, 2018; Weile et al., 1996), where predefined 16 virtual materials were preferred, MRA designs were performed by means of either single objective (Asi and Dib, 2010; Goudos, 2009; Goudos and Sahalos, 2006a; Kern and Werner, 2003; Michielssen et al., 1993; Ranjan 
et al., 2018; Roy et al., 2016, 2015; Toktas et al., 2018) or multi-objective (MO) approaches (Goudos and Sahalos, 2006b; Jiang et al., 2009; Toktas et al., 2018; Weile et al., 1996). In the MO approach, both thickness and reflection coefficients are optimized simultaneously, while in single objective optimization all objectives are combined in a single objective function by means of weight coefficients (Goudos, 2009). In addition, a new double-stage method has recently been introduced that optimizes the total thicknesses (TT) of layers separately from number \& sequence of the layers (NSL) (Yigit and Duysak, 2019a) . Thanks to the double-stage approach, negative effects of sudden changes in layer sequence has been prevented (Yigit and Duysak, 2019a). However, similar to other single or MO approaches (Asi and Dib, 2010; Goudos, 2009; Goudos and Sahalos, 2006a, 2006b; Jiang et al., 2009; Kern and Werner, 2003; Michielssen et al., 1993; Ranjan et al., 2018; Roy et al., 2016, 2015; Toktas et al., 2019, 2018; Weile et al., 1996), in (Yigit and Duysak, 2019a) the reflection coefficients for Transverse Electric (TE) and Transverse Magnetic (TM) polarizations were combined in a single objective function. Satisfactory results can be achieved by combining different objective functions at the beginning of the algorithm, but in some special cases, it is inevitable that MO optimization is required for compromises between objectives. For example, in some cases, it may be desirable to minimize the maximum reflection coefficient by sacrificing TT. In this case, MO optimization approaches are inevitable.

In this study, three different objectives (TT, reflection coefficients for TE and TM polarizations) for fully optimized MRA design are synchronously minimized by means of Pareto-integrated triple objective ABC (TOABC) algorithm. In MRA design problem, it is not a reasonable approach to set a minimum or maximum objective for the number of layers (NL). As it can be seen from (Yigit and Duysak, 2019a), much thinner structures can be designed by using more layers. Therefore, in this study, NSL is simultaneously optimized with thicknesses. A graphical user interface (GUI) is designed on the MATLAB platform to easily obtain possible solutions on the Pareto front. The effectiveness of the method is demonstrated by using both 16 predefined virtual materials (Michielssen et al., 1993) and up-to-date 21 real materials(Yigit and Duysak, 2019a). The designed structures are compared with the similar multi-layer radar absorbers in the literature(Ranjan et al., 2018). 3 different designs (for TE/TM modes) effective at the frequency band of $2-8 \mathrm{GHz}, 12-18 \mathrm{GHz}$ and $2-18 \mathrm{GHz}$ at the angles of incident between $0^{\circ}$ and $60^{\circ}$ are optimized. In addition, 4 different MRA structures are also designed, which consist entirely of real materials, operating across the entire band from 2 to $18 \mathrm{GHz}$ for all angles of incident between $0^{\circ}$ and $60^{\circ}$.

The physical model of the MRA design and the basic theory of the $\mathrm{ABC}$ algorithm are briefly presented in the next section. In Section III, the detailed information of TO-ABC is presented. The implementation of the TO-ABC algorithm and optimized MRA designs are given in Section IV. The concluding remarks and possible further studies are mentioned in the conclusion section.

\section{PHYSICAL MODEL of MRA STRUCTURE and ABC ALGORITHM}

\subsection{Physical Model of MRA Structure}

A basic MRA structure consists of different materials coated on a perfect electric conductor. Each layer is defined by different electrical properties and different thickness. The main purpose of the MRA design is to determine the NSL and TT, to minimize the back scattered energy for TE \& TM polarizations for desired angle of incidences and frequencies. While the EM wave travels through each layer, it is exposed to the transmission, the absorption and the reflection effects in the each layer. Therefore, the total surface reflection of the MRA depends on the alignment of the entire structure. Since the detailed formulation of MRA design problem was given in many studies (Asi and Dib, 2010; Goudos, 2009; Goudos and Sahalos, 2006a, 2006b; Jiang et al., 2009; Kern and Werner, 2003; Michielssen et al., 1993; Ranjan et al., 2018; Roy et al., 2016, 2015; Toktas et al., 2019, 2018; Weile et al., 1996; Yigit and Duysak, 2019a), in this study only the basic equations are given and critical points are indicated. In the polarizationinsensitive MRA design, the back-scattered energy must be minimized in both TE and TM polarizations along with the TT of the MRA. Therefore, three objective functions

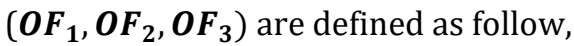

$$
\begin{gathered}
\operatorname{minimize} \mathrm{OF}_{1}=\operatorname{maximum}\{20 \log 10(R T E)\} \\
\text { minimize } \mathrm{OF}_{2}=\operatorname{maximum}\{20 \log 10(R T M)\} \\
\text { minimize } \mathrm{OF}_{3}=T T
\end{gathered}
$$

where $\boldsymbol{R} \boldsymbol{T} \boldsymbol{M}$ and $\boldsymbol{R T} \boldsymbol{E}$ are the reflection coefficient matrices, which includes the TM and TE polarizations corresponding to each of the defined angle of incidence and the frequency values. For an MRA structure with $\boldsymbol{M}$ $\left(\mathrm{M}^{\text {th }}\right.$ layer is perfect electric conductor) layer, the RTE and RTM are defined as follows (Yigit and Duysak, 2019a),

$$
\begin{aligned}
& {R_{i}}^{T E}=\frac{r_{i}^{T E}+R_{i+1}^{T E} e^{-2 j k_{i+1} d_{i+1}}}{1+{r_{i}}^{T E} R_{i+1}^{T E} e^{-2 j k_{i+1} d_{i+1}}} \\
& R_{i}^{T M}=\frac{r_{i}^{T M}+R_{i+1}{ }^{T M} e^{-2 j k_{i+1} d_{i+1}}}{1+r_{i}{ }^{T M} R_{i+1}{ }^{T M} e^{-2 j k_{i+1} d_{i+1}}}
\end{aligned}
$$

where $\boldsymbol{d}_{\boldsymbol{i}}$ corresponding to the thickness of each layer. The $\boldsymbol{r}_{\boldsymbol{i}}^{\boldsymbol{T} \boldsymbol{E}}$ and $\boldsymbol{r}_{\boldsymbol{i}}^{\boldsymbol{T} \boldsymbol{M}}$ are given as follows,

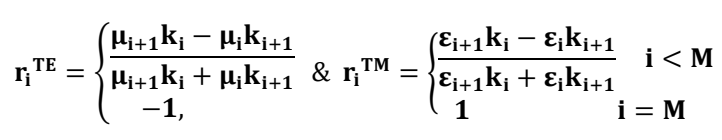

According to the angle of incidence $\boldsymbol{\theta}^{\circ}$, the wave number $\boldsymbol{k}_{\boldsymbol{i}}$, of the $\boldsymbol{i}^{\boldsymbol{t h}}$ layer is defined as,

$$
k_{i}=2 \pi f \sqrt{\mu_{i} \varepsilon_{i}-\mu_{0} \varepsilon_{0} \sin ^{2}(\theta)}
$$

In (4), while $\boldsymbol{\mu}_{\boldsymbol{i}}$ and $\boldsymbol{\varepsilon}_{\boldsymbol{i}}$ are the frequency(f) depended complex permeability and permittivity of the materials, $\boldsymbol{\mu}_{\mathbf{0}}$ and $\boldsymbol{\varepsilon}_{\mathbf{0}}$ are permeability and permittivity of free space, 
respectively (Yigit and Duysak, 2019a). According to the (2) and (3), the TT of the structure is defined as follow,

$$
T T=\sum_{i=1}^{M-1} d_{i}
$$

\subsection{Single-Objective ABC Algorithm for MRA Design}

Natural inspired metaheuristic optimization techniques can produce satisfactory results which are close to the optimum result for nonlinear problems. ABC is an efficient algorithm inspired by the nectar search activities of honey bee swarms (Karaboga and Basturk, 2007). In the algorithm, the number of food sources (NS) specify the potential solutions of the problem, while the amount of nectar in the sources corresponds to fitness value of the solution. The details of the algorithm developed by modeling the behavior of employed, onlooker and scout bees are given in (Yigit and Duysak, 2019a). The changes made in these three phases in the MO-ABC algorithm are explained in detail in the next section.

\section{MO-ABC ALGORITHM}

\subsection{Multi -Objective Optimization}

In the single-objective optimization, optimum results can be obtained by comparing only the single value of the objective function. However, if there is more than one objective function, the problem is defined as a MO optimization problem and all objective functions are simultaneously optimized. The mathematical expression of MO optimization problem can be defined as follows.

$$
\begin{gathered}
\min F(\chi)=\left[f_{1}(\chi), f_{2}(\chi), \ldots \ldots, f_{\Pi}(\chi)\right] \\
\chi=\left[x_{1}, x_{2}, \ldots \ldots, x_{u}\right] \in S
\end{gathered}
$$

where $\boldsymbol{\Pi}, \boldsymbol{\chi}$ and $\boldsymbol{S}$ are the number of the objective functions, $\boldsymbol{u}$ dimensional decision variable vector and the search space, respectively. The solution of the multiobjective optimization problem is generally represented by a Pareto optimal set (Deb et al., 2002; Huo et al., 2015). For the minimization problems, if at least one objective value of the solution $\chi$ is less than the other solution $\chi^{\prime}$, and all objective values of $\chi^{\prime}$ aren't less than $\chi$, then $\chi$ dominates $\chi^{\prime}$. However, if no solution dominates the solution $\chi^{\prime}$, then $\chi^{\prime}$ is called as the nondominated or Pareto-efficient solution. All Pareto solutions constitute elite solution (ES) set which are the possible solutions of the MO problem.

\subsection{Multi-Objective ABC Algorithm}

The MO-ABC algorithm includes four phase; initialization, employed bees, onlooker bees and scout bees. The flow chart of the algorithm is given in the Fig.1.

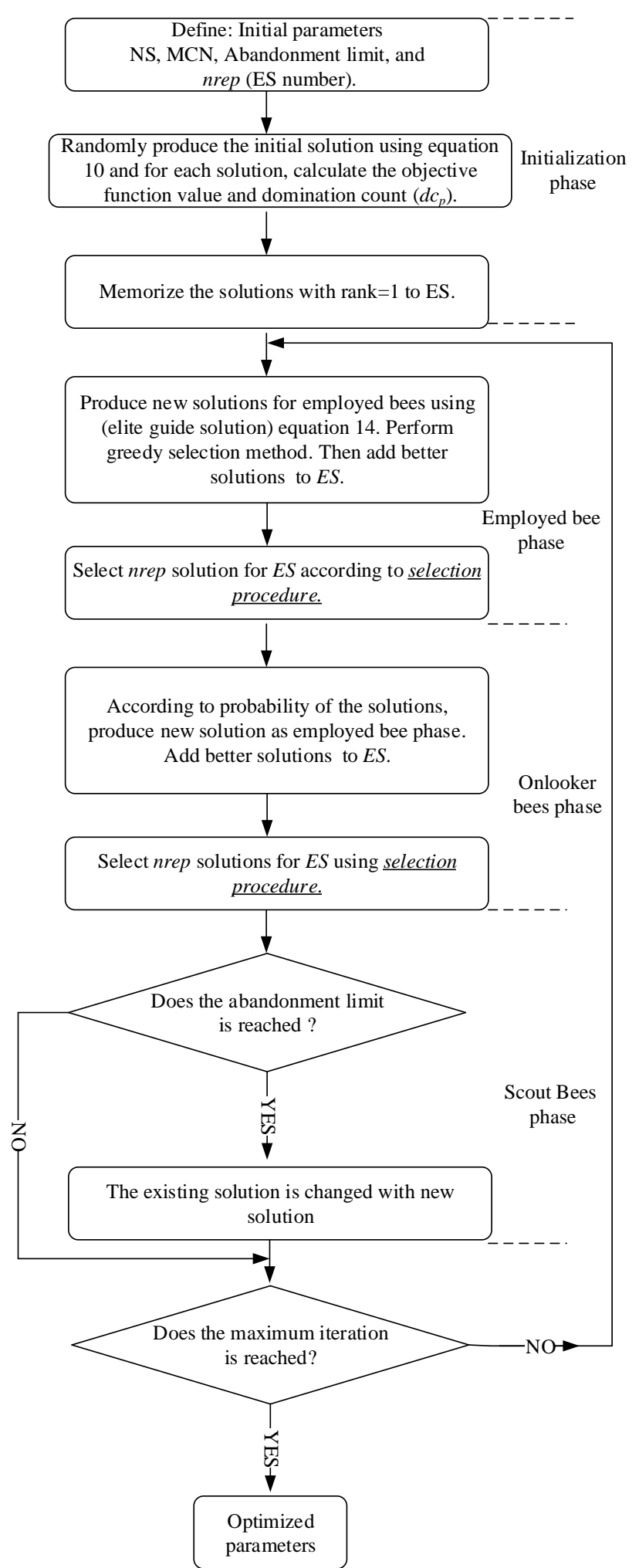

Figure 1. The flow chart of MOABC

1) Initialization

The population $\boldsymbol{P}$ of the bee swarm is defined as follows,

$$
P=\left\{\chi_{1}, \chi_{2}, \ldots, \chi_{N S}\right\}
$$

where $\boldsymbol{N} \boldsymbol{S}$ is the number of food source. The initial food sources $\chi_{p}$ are randomly generated by (10),

$$
\chi_{p}=l b^{d}+\operatorname{rand}(0,1)\left(u b^{d}-l b^{d}\right)
$$


where $\boldsymbol{p} \in\{\mathbf{1}, \mathbf{2}, \ldots ., \boldsymbol{N} \boldsymbol{S}\}$ and $\boldsymbol{d} \in\{\mathbf{1}, 2, \ldots \ldots, \boldsymbol{u}\}$ are the solution vector index and dimension of $\chi_{p}$, respectively. $\boldsymbol{l} \boldsymbol{b}$ and $\boldsymbol{u} \boldsymbol{b}$ are lower and upper bound of solution vectors.

\section{- Determination of elite solutions.}

In MO optimization, there are more than one objective function, so it is not simple to determine the nondominated solutions. In this study, in order to sort and select the ES, fast nondominated sorting (Deb et al., 2002) requiring the less computations, is preferred.

For each solution, two parameters are calculated, these are;

1. Domination count, $\boldsymbol{d} \boldsymbol{c}_{\boldsymbol{p}}$ : the number of solutions which dominate the solution $\boldsymbol{p}$,

2. $\boldsymbol{S}_{\boldsymbol{P}}$ : Set of the solutions dominated by solution $\boldsymbol{p}$.

In fast nondominated sorting method, the initial values of $\boldsymbol{d} \boldsymbol{c}_{\boldsymbol{p}}$ of all solutions in population are defined as zero. For each solution $\boldsymbol{p}$ in the population $\boldsymbol{P}$ is then compared with every other solution $\boldsymbol{q}$ in $\boldsymbol{P}$. If the solution $\boldsymbol{p}$ is better than $\boldsymbol{q}$, then $\boldsymbol{S}_{\boldsymbol{P}}=\boldsymbol{S}_{\boldsymbol{P}} \cup\{\boldsymbol{q}\}$, otherwise; $\boldsymbol{d} \boldsymbol{c}_{\boldsymbol{p}}=$ $\boldsymbol{d} c_{p}+1$. If $\boldsymbol{d} c_{p}=0, d c_{p} \cdot \operatorname{rank}=1$. Otherwise, $\boldsymbol{d} c_{p}$ corresponding to each member $(\boldsymbol{q})$ of $\boldsymbol{S}_{\boldsymbol{P}}$ is reduced by one. When $\boldsymbol{d} \boldsymbol{c}_{\boldsymbol{p}}$ of $\boldsymbol{q}$ is zero, it is saved to the new $\boldsymbol{Q}$ list. This $\boldsymbol{Q}$ list constitutes the second nondominated front. This process continues until all front is determined (Deb et al., 2002). In this study, ES consists of the first nondominated front as illustrated in Fig. 2(a).

\section{- Crowding Distance}

The crowding-distance is obtained according to each objective function value of the Pareto points. For each objective function, the distance values of the largest and smallest points are assumed as an infinite. The initial values of the distances of all solutions are assigned as zero and then the distances of each solutions for each objective function are updated as follows (Deb et al., 2002).

$$
d i s t_{m, j}=d i s_{m, j}+\frac{f_{m, j+1}-f_{m, j-1}}{\max \left(f_{m}\right)-\min \left(f_{m}\right)}
$$

where dist $_{\mathbf{m}, \mathbf{j}}$ denotes distance of $\mathrm{j}^{\text {th }}$ solution of $\mathrm{m}^{\text {th }}$ objective function as illustrated in Fig. $2 \mathrm{~b}$.

The exact crowding-distance vector $\left(\mathbf{C d}_{\mathbf{j}}\right)$ is obtained by summing the individual distance values corresponding to each objective as follows,

$$
C d_{j}=\sum_{\mathrm{m}=1}^{\Pi} d i s t_{m, j}
$$

\section{- $\quad$ Selection procedure}

The number of ES can be more than the desired number of archive, nrep. Therefore, population selection strategy must be applied to select solutions to constitute ES. Since the ranks of the all solutions in ES are equal to 1 , the solutions with the higher distance should be selected.
For this purpose, the crowding distance values (Cd) of all solutions are sorted in descending form and the first nrep solutions are selected.

\section{2) Employed Bee Phase}

In the employed bee phase, bees search new food sources to improve the existing solution. In the original ABC algorithm(Karaboga and Basturk, 2007), a new food source $\chi^{\prime}$ is generated by using (13) for each employed bee sources $(\mathbf{n}=\mathbf{1} \ldots \mathbf{N S})$,

$$
\chi_{n r}^{\prime}=\chi_{n r}+\phi\left(\chi_{n r}-\chi_{k r}\right)
$$

where $\chi_{\mathbf{k r}}$ is neighbour solution of $\chi_{\mathbf{n r}}$ corresponding to the randomly generated $\mathbf{r} \in[\mathbf{1}, \mathbf{u}]$.

Original $A B C$ algorithm don't use the ES or best solution. However, to effectively improve the solutions in MO-ABC, the new solutions must be updated based on the ES (Huo et al., 2015). In the second phase of the MO$\mathrm{ABC}$ algorithm, a new food source is produced by the following equation (Huo et al., 2015),

$$
\chi_{n r}^{\prime}=\chi_{n r}+\phi\left(\chi_{n r}-\chi_{k r}\right)+\psi\left(y_{n r}-\chi_{k r}\right)
$$

where $\phi$ and $\psi$ are random numbers in the ranges of $[-1,1]$ and $[0,2]$, respectively. $\mathbf{y}_{\mathbf{n r}}$ denotes the randomly selected solution from $\mathbf{E S}$.

The choice between the existing and new solutions is determined by means of the greed selection. If the existing solution is worse than the new solution, the abandonment counter is reset and $\mathbf{E S}=\mathbf{E S} \cup\left\{\boldsymbol{\chi}_{\mathbf{n}}^{\prime}\right\}$; otherwise, the counter is increased. After all employed bees search new food source, ES is updated by performing the selection procedure.
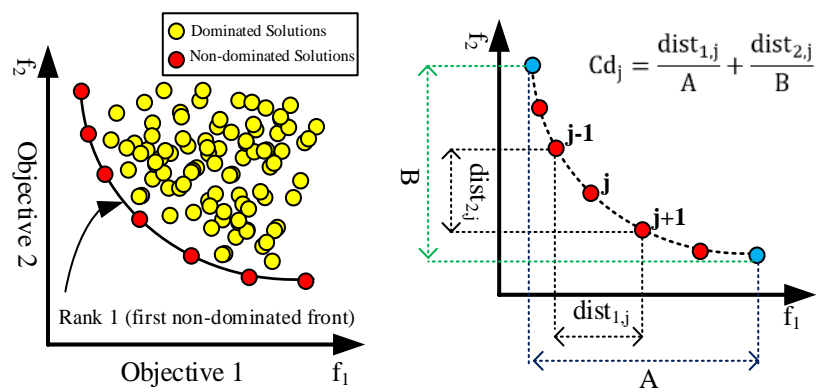

Figure 2. Illustration of the Pareto front points; a) Dominated and non-dominated solutions, Determination of Crowded distances

\section{3) Onlooker Bee Phase}

In the onlooker bee phase, in order to calculate the selection probability value, the fitness value of each solution must be calculated. For a single objective function, the fitness value is calculated as follows (Karaboga and Basturk, 2007)

$$
f i t\left(\chi_{n}\right)=\frac{1}{1+f\left(\chi_{n}\right)}
$$

However, in the MO optimization, since the number of objective functions is more than one, (15) is not applicable. Thus, the fitness function based on 
normalization should be used. The normalization calculation is as follows (Huo et al., 2015),

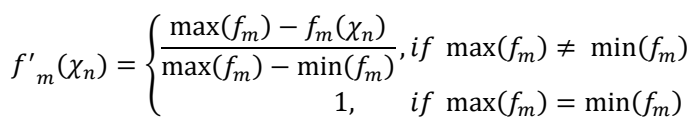

The $\boldsymbol{f} \boldsymbol{i t}\left(\chi_{n}\right)$ is calculated as following equation (Huo et al., 2015)

$$
f i t\left(\chi_{n}\right)=\frac{1}{\Pi} \sum_{m=1}^{\Pi}{f^{\prime}}_{m}\left(\chi_{n}\right)
$$

The selection probability is calculated as follows,

$$
\operatorname{prob}_{n}=\frac{0.9 f i t\left(\chi_{n}\right)}{\max _{n=1}^{N S} f i t\left(\chi_{n}\right)}+0.1
$$

Then, each of the onlooker bees searches the new food source depending on the selection probability. If randomly generated number in the range of $[\mathbf{0 , 1}]$ is greater than $\boldsymbol{p r o b} \boldsymbol{b}_{n}$, the new solution will be produced using (14) and the steps in the employed bee phase are applied.

\section{4) Scout Bee Phase}

In this phase, if a solution is not improved when the abandonment limit is reached, the existing solution is changed. For this purpose, the existing employed bee is transformed to the scout bee and a new food source is generated by (10). Thus, the algorithm continues to run until the maximum cycle number $(\mathrm{MCN})$ or it reaches a predefined stop criteria.

\section{IMPLEMENTATION OF TO-ABC ALGORITHM}

The TO-ABC method for MRA design consists of 3 main parts as seen in Fig. 3. The objective functions of the algorithm are defined as given in (1).

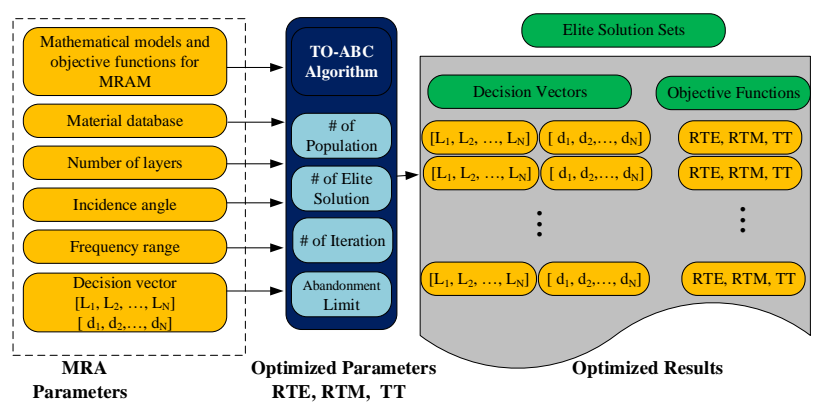

Figure 3. Implementation of TO-ABC to design of MRA

The parameters of the MRA structure and TO-ABC algorithm are defined as given in Fig. 3. Since the minimum layer thicknesses are defined as 0 , at the end of the optimization the layers with zero thicknesses are removed from the sequence. Thus, the ES are listed as shown in the Fig. 3 and optimized results are obtained.

\subsection{Graphical User Interface for MRA Design}

Dozens of studies have been done for the MRA design in the last quarter century. Each of these studies aimed to develop a part of the design problem. In this study, a fully optimized MRA design method is presented for the first time. However, since there are many parameters to consider in MRA design, an easily accessible interface is needed for real applications. Therefore, in this study, a GUI is developed for a fully optimized MRA design. As seen in Fig. 4 the GUI has 5 different parametric inputs such as Frequency, Angle of Incidence, MRA, Optimization and Material List. After these inputs have been defined, the program is run by clicking the "Start MRA Design" button. Once the optimization is completed, the optimal results determined on the Pareto front are illustrated in the GUI, while the corresponding RTE, RTM and TT values are listed in the "Elite Solution" section. In GUI, the operator selects the desired result according to the trade-off between RTE, RTM and TT. The NSL and thicknesses of layers corresponding to the selection of the operator are given in the "Layer sequence \& Thickness" section and the reflection coefficients for TE and TM polarizations are plotted depending on the angle of incidence. Thus, with the developed GUI, MRA designers can easily access the results they need.

\subsection{Fair Comparison Criterias and Comparison Data}

Several studies have been conducted for MRA design so far and the superiority of the proposed methods in each study have been compared with previous ones. In order to make a fair comparison, the designed MRAs should be compared based on the same parameters. These parameters can be summarized as follows.

1. When comparing the two designs, it is not fair to consider only the maximum reflection coefficients and to ignore thicknesses. Because it is obvious in MRA design that maximum reflection coefficient decreases with increasing thickness.

2. It is also not true to compare broad-band wide-angle designs for a single type of polarization. For example, while the maximum reflection coefficient for TE polarization is minimized, TM increases. Therefore, the reflection coefficients for $\mathrm{TE}$ and $\mathrm{TM}$ polarizations need to be optimized simultaneously.

3. When making comparisons, optimizing both designs for the same angle of incidence is another important consideration. For example, it is a not fair comparison to compare an MRA structure which is optimized between $0^{\circ}$ and $40^{\circ}$ with another design which is optimized between $0^{\circ}$ and $60^{\circ}$. Because, it is an expected result that the MRA optimized for $60^{\circ}$ has worse RTE, RTM and TT values than the MRA optimized for $40^{\circ}$.

4. Finally, when presenting the performances of the designs, it is not a consistent method to give the average values of the reflection coefficients relative to the all frequency band and angle of incidence. Because, if the design has a very low reflection coefficient at any angle or frequency, it significantly reduces the average value and affects the general information about other values.

Therefore, these issues should be taken into consideration in order to make a rational assessment. For example, the designs optimized between $0-40^{\circ}$ in (Toktas et al., 2019) were compared to those given in 
references(Ranjan et al., 2018; Roy et al., 2015). However, since the designs given in (Roy et al., 2015) were optimized only for normal incidence, their performances at $40^{\circ}$ are inherently worse than the (Toktas et al., 2019). It is also unfair to compare the designs in (Toktas et al., 2019) with the designs in (Ranjan et al., 2018) , as the MRAs in (Ranjan et al., 2018) were optimized to operate between $0^{\circ}$ and $60^{\circ}$.

Based on these 4 criteria, in this study, the designs optimized with 16 virtual materials are compared to those in (Ranjan et al., 2018). Since the formulation (2)\&(3) was verified with computer simulation technology (CST) in the previous study(Yigit and Duysak, 2019a), the CST results are not given in this study.

\subsection{Designed Fully Optimized MRAs with 16 Virtual Materials}

To show the success and practice usage of the TO$\mathrm{ABC}$, virtual materials listed in (Michielssen et al., 1993) are selected as the material list in the GUI. Three types of MRAs effective in the frequency ranges of 2-8 (Des1), 1218 (Des2) and 2-18 GHz (Des3) are optimized. The angles of incidence are changed between $0^{\circ}$ and $60^{\circ}$. These are compared with those in(Ranjan et al., 2018). All design parameters except the frequency band are selected as shown in Fig. 4. While plotting the reflection coefficients, to obtain high-resolution graphics, the resolutions of frequency and angle are determined as $0.1 \mathrm{GHz}$ and $1^{0}$, respectively. As seen in Table 1, at the end of the TO-ABC algorithm, different MRA structures having different NL and sequences are optimized. As shown in Fig. 4, although the number of ES are identified as 50, a total of 19 possible solutions are found and 7 of them are given in Table 1. It should be noted that the RTE and RTM values given in all Tables are the maximum values between all angles of incidence $\left(\boldsymbol{\theta} \in\left[\mathbf{0}^{\circ}, \mathbf{6 0}^{\circ}\right]\right)$ and frequencies. The 3D plots of RTE and RTM according to angle of incidence and the frequency are illustrated in Fig. 4. When these solutions are compared with the results given in the (Ranjan et al., 2018), it is seen that the highlighted solution 12 in Table 1 is similar to the design in the (Ranjan et al., 2018).

However, although there is a difference of $0.02 \mathrm{~dB}$ in RTE, RTM and TT values are lower than those found in [13]. When the other values in Table 1 are examined, it is seen that there are much thinner designs with much lower RTM values than the solution 12. However, it should be noted that RTE values of the other solutions are higher than solution 12.When the solution 13 of Des2 is compared with the respective MRA(Ranjan et al., 2018) in Table 2, it can be seen that both TT, RTE and RTM of the MRA are lower than the(Ranjan et al., 2018). This result clearly demonstrates the success of the proposed method. Furthermore, due to the nature of the MO approach, lower reflection coefficients can be obtained by sacrificing thickness or much finer MRAs can be chosen from Table 2 by compromising from reflection coefficient. Considering Table 3, where the results of Des3 are listed, it can be seen that all the ES consist of 4 layers and most of them have same sequence except from third layer. Furthermore, when the solution 16 in Table 3 is compared with (Ranjan et al., 2018), it is seen that although they have the same sequence, both the RTE, RTM and TT values of the solution 16 are much better than the (Ranjan et al., 2018). This result is a clear indication of the necessity of the triple objective approach in MRA design.

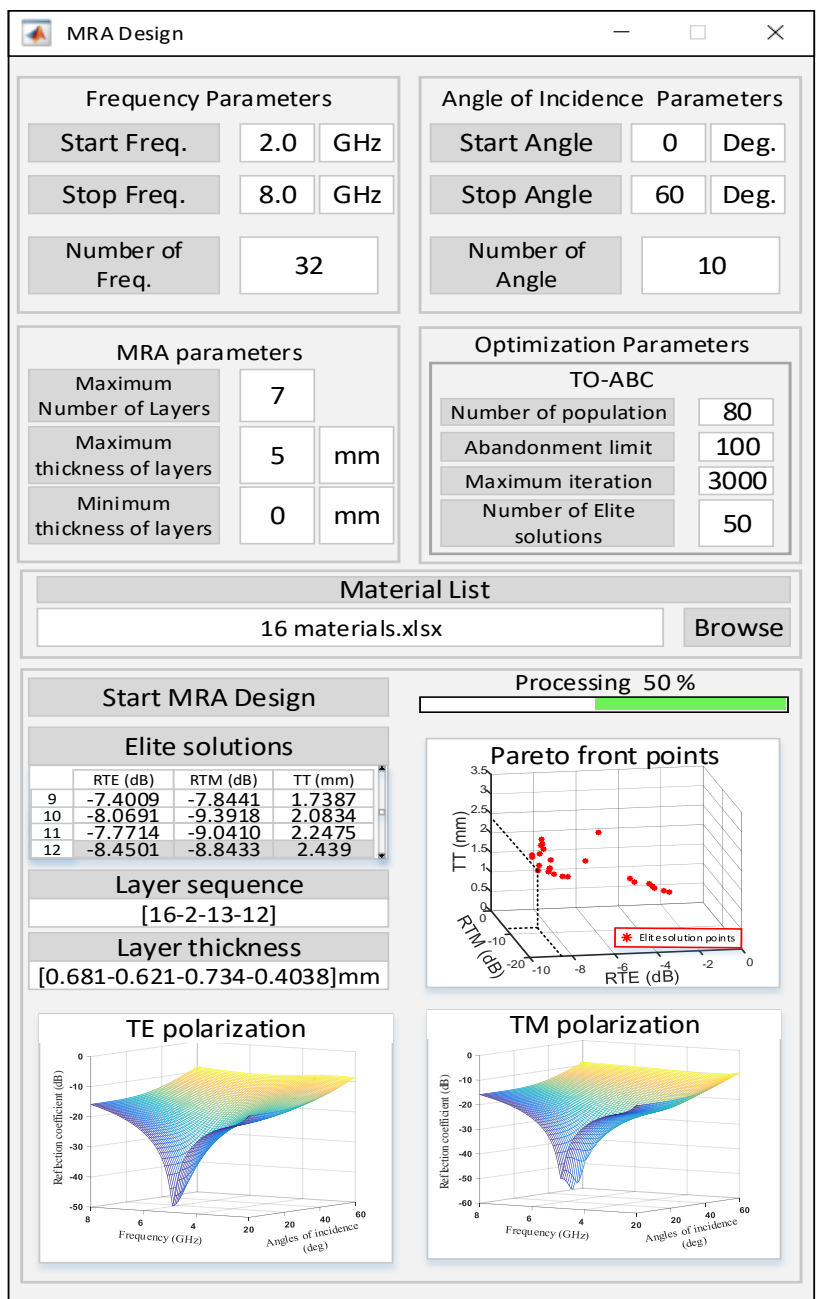

Figure 4. The GUI for fully optimized MRA design

Table 1. The results corresponding to some of the pareto points obtained by TO-ABC algorithm for Des1 (2-8 ghz)

\begin{tabular}{|c|c|c|c|c|c|c|c|c|}
\hline & Layer sequence & $\mathrm{d} 1$ & $\mathrm{~d} 2$ & $\mathrm{~d} 3$ & $\mathrm{~d} 4$ & RTE & RTM & TT \\
\hline 6 & [16-1-9] & 0.569 & 0.332 & 0.838 & & -7.401 & -7.844 & 1.739 \\
\hline 7 & [16-2-10-11] & 0.572 & 0.302 & 0.415 & 0.560 & -7.554 & -9.541 & 1.849 \\
\hline 8 & {$[16-2-10-9]$} & 0.650 & 0.300 & 0.373 & 0.581 & -7.768 & -9.045 & 1.903 \\
\hline 9 & [16-11-2-15] & 0.486 & 0.345 & 0.877 & 0.300 & -8.056 & -7.834 & 2.008 \\
\hline 10 & [16-2-9-11] & 0.656 & 0.447 & 0.417 & 0.563 & -8.069 & -9.392 & 2.083 \\
\hline 11 & [14-2-13] & 0.629 & 1.006 & 0.612 & & -7.771 & -9.041 & 2.248 \\
\hline 12 & [16-2-13-12] & 0.681 & 0.621 & 0.734 & 0.404 & -8.450 & -8.843 & 2.440 \\
\hline \multicolumn{9}{|c|}{ Data from (Ranjan et al., 2018) } \\
\hline & Layer sequence & $\mathrm{d} 1$ & d2 & d3 & $\mathrm{d} 4$ & RTE & RTM & TT \\
\hline & [16-2-13-12] & 0.701 & 0.657 & 0.707 & 0.383 & -8.476 & -8.570 & 2.449 \\
\hline
\end{tabular}


Table 2. The results corresponding to some of the pareto points obtained by TO-ABC algorithm for Des2 (8-12 GHz)

\begin{tabular}{|c|c|c|c|c|c|c|c|c|}
\hline & Layer sequence & $\mathrm{d} 1$ & $\mathrm{~d} 2$ & d3 & $\mathrm{d} 4$ & RTE & RTM & TT \\
\hline 8 & [16-2] & 0.364 & 0.286 & & & -6.668 & -12.685 & 0.650 \\
\hline 9 & [16-8] & 0.376 & 0.370 & & & -7.050 & -12.879 & 0.746 \\
\hline 10 & [16-2-5-6] & 0.376 & 0.276 & 0.040 & 0.124 & -7.742 & -9.050 & 0.817 \\
\hline 11 & {$[16-8-6]$} & 0.376 & 0.308 & 0.250 & & -7.776 & -10.878 & 0.935 \\
\hline 12 & [16-8-16] & 0.259 & 0.651 & 0.136 & & -8.021 & -11.531 & 1.046 \\
\hline 13 & [16-8-12-6] & 0.376 & 0.560 & 0.014 & 0.162 & -8.543 & -10.484 & 1.113 \\
\hline 14 & [16-8-6] & 0.373 & 0.634 & 0.250 & & -9.302 & -9.622 & 1.256 \\
\hline \multicolumn{9}{|c|}{ Data from (Ranjan et al., 2018) } \\
\hline & Layer sequence & d1 & $\mathrm{d} 2$ & d3 & $\mathrm{d} 4$ & RTE & RTM & TT \\
\hline & {$[16-7-3-8]$} & 0.358 & 0.414 & 0.130 & 0.293 & -8.510 & -9.940 & 1.195 \\
\hline
\end{tabular}

Table 3. The results corresponding to some of the pareto points obtained by TO-ABC algorithm for Des3 (2-18 GHz)

\begin{tabular}{|c|c|c|c|c|c|c|c|c|}
\hline & Layer sequence & d1 & $\mathrm{d} 2$ & $\mathrm{~d} 3$ & $\mathrm{~d} 4$ & RTE & RTM & TT \\
\hline 12 & [16-7-3-14] & 0.340 & 1.227 & 0.379 & 0.501 & -6.714 & -7.277 & 2.447 \\
\hline 13 & {$[16-7-3-14]$} & 0.340 & 1.036 & 0.731 & 0.385 & -6.768 & -7.854 & 2.492 \\
\hline 14 & {$[16-7-3-14]$} & 0.340 & 1.183 & 0.631 & 0.425 & -6.899 & -7.782 & 2.579 \\
\hline 15 & {$[16-7-4-14]$} & 0.340 & 1.300 & 0.568 & 0.445 & -6.972 & -8.448 & 2.653 \\
\hline 16 & [16-7-5-14] & 0.349 & 1.245 & 1.000 & 0.319 & -7.444 & -8.466 & 2.912 \\
\hline 17 & [16-6-3-14] & 0.340 & 1.232 & 1.063 & 0.367 & -7.333 & -8.183 & 3.002 \\
\hline 18 & [16-7-3-15] & 0.340 & 1.264 & 1.094 & 0.367 & -7.150 & -8.658 & 3.065 \\
\hline \multicolumn{9}{|c|}{ Data from (Ranjan et al., 2018) } \\
\hline & $\begin{array}{c}\text { Layer sequence } \\
{[16-7-5-14]}\end{array}$ & $\begin{array}{c}\mathrm{d} 1 \\
0.348\end{array}$ & $\begin{array}{c}\mathrm{d} 2 \\
1.246\end{array}$ & $\begin{array}{c}\mathrm{d} 3 \\
1.002\end{array}$ & $\begin{array}{c}\mathrm{d} 4 \\
0.326\end{array}$ & $\begin{array}{c}\text { RTE } \\
-7.400\end{array}$ & $\begin{array}{c}\text { RTM } \\
-8.300\end{array}$ & $\begin{array}{c}\text { TT } \\
2.920\end{array}$ \\
\hline
\end{tabular}

\subsection{Designed MRAS with Real Materials}

After the importance of TO-ABC approach in the fully optimized MRA design has been proved by the use of predefined virtual materials, real MRA structures have been designed with much more limited real materials. For this purpose, a new material pool of 21 fabricated materials presented in the literature(Yigit and Duysak, 2019a) are used. The compositions of the materials and measured operating frequencies are given in Table 4 . The frequency dependent complex permeabilities $\mu_{i}$ and permittivities $\boldsymbol{\varepsilon}_{\boldsymbol{i}}$ of these 21 up-to-date materials can be downloaded from (Yigit and Duysak, 2019a, 2019b). 4 types of MRA structures labeled as Des4 (2-8 GHz), Des5 $(8.2-12.4 \mathrm{GHz})$, Des6 (12-18GHz) and Des7 $(2-18 \mathrm{GHz})$ are optimized at wide-angle $\left(0^{\circ}-60^{\circ}\right)$. The layer properties and labels of the selected designs are listed in Table 5. In GUI software, the control parameters of the optimization process such as maximum number of layers, abandonment limit, maximum iteration number, population number and maximum number of elite solution are selected as 10,100,3000, 100 and 100, respectively. The maximum and minimum layer thicknesses are defined as $4 \mathrm{~mm}$ and $0 \mathrm{~mm}$, respectively. Thus, at the end of the optimization, the layers having a thickness of $0 \mathrm{~mm}$ is removed from the structure. In these designs, since the used real materials are limited, TT is ignored when choosing between the ES and the solutions with minimum reflection coefficient are presented. Des4, Des5, Des6 and Des7 are selected by considering both
RTE and RTM values. While some designs have lower RTM values, these designs have higher RTE values than other designs.

Although the reflection coefficients of the designed MRAs can be simply obtained by using $(2,3)$, for an easier assessment, RTE and RTM corresponding to the each $10^{\circ}$ angle of incidences are presented in Table 5. As seen in Table 6, 7, 8 and 9, 7 design are given in each table. As shown in Table 6, the RTE and RTM of solution 33 of Des5 are -6.29 and $-11.79 \mathrm{~dB}$ for whole angles of incidence and the frequencies. In the initial parameters of the TO-ABC algorithm, this value can be selected larger, so that thicker, but better MRA structures can be designed. Since the all materials given in Table 4 can be used in Des5, much more successful MRA structures are designed than the Des4. As shown in Table 7, the TT of solution 20 is $4.058 \mathrm{~mm}$, although it has an absorption capability of $87 \%$ for all frequencies and all angles of incident between $0^{0}-60^{\circ}$. When the solution 24 given in Table 7 is observed, it is realized that the TT increases but the maximum reflection coefficients considerably decreases. In Des6, 8 materials given in the Table 4 are used. The ES obtained after TO-ABC are given in Table 8. Although all solutions have the same layer sequence, they have different thickness and reflection coefficient values. Among these solutions, the solution 15 with the lowest RTE value is selected and the RTE and RTM values are seen in Table 5. 
Table 4. Up-to-date materials listed in literature (Yigit and Duysak, 2019a, 2019b)

\begin{tabular}{|c|c|c|}
\hline Material number & Fabricated Materials & $\begin{array}{l}\text { Measured Frequency } \\
\text { range (GHz) }\end{array}$ \\
\hline 17 & $\begin{array}{l}\text { Dispersed Alinco }\left(\mathrm{Fe}_{49.7} \mathrm{Co}_{24} \mathrm{Ni}_{14} \mathrm{Al}_{8} \mathrm{Cu}_{3} \mathrm{Nb}_{1} \mathrm{Ti}_{0.3}\right) \text { powder in paraffin wax. Fraction } \\
\text { of powder is } 50 \%\end{array}$ & $2-18$ \\
\hline 18 & Dispersed Alinco powder in paraffin wax. Fraction of powder is $60 \%$ & $2-18$ \\
\hline 19 & $\mathrm{Ba}_{0.85} \mathrm{Sm}_{0.15} \mathrm{Co}_{2} \mathrm{Fe}_{16} \mathrm{O}_{27}$ hexaferrite & $2-18$ \\
\hline 20 & Pure porous carbon, obtained through the pyrolysis of pure ZIF-67 & $2-18$ \\
\hline 21 & 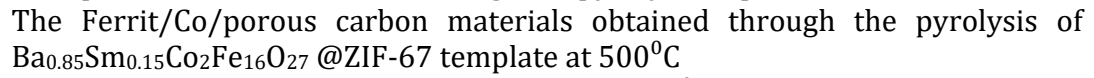 & $2-18$ \\
\hline 22 & $\ldots \mathrm{Ba}_{0.85} \mathrm{Sm}_{0.15} \mathrm{Co}_{2} \mathrm{Fe}_{16} \mathrm{O}_{27} @ \mathrm{ZIF}-67$ template at $550^{\circ} \mathrm{C}$ & $2-18$ \\
\hline 23 & $\ldots \mathrm{Ba}_{0.85} \mathrm{Sm}_{0.15} \mathrm{Co}_{2} \mathrm{Fe}_{16} \mathrm{O}_{27} @ \mathrm{ZIF}-67$ template at $650^{\circ} \mathrm{C}$ & $2-18$ \\
\hline 24 & Carbon coated nickel nanocapsule & $2-18$ \\
\hline 25 & $\begin{array}{l}\text { U-type hexaferrite } \\
\left.\mathrm{Ba}_{4}\left(\mathrm{Co}_{1-3 \mathrm{x}} \mathrm{Cr}_{\mathrm{x}}\right)_{2} \mathrm{Fe}_{36} \mathrm{O}_{60}\right) \text { for different values of } x\end{array}$ & $8.2-12.4$ \\
\hline 26 & $\begin{array}{l}\text { U-type hexaferrite } \\
\left.\mathrm{Ba}_{4}\left(\mathrm{Co}_{1-3 \mathrm{x}} \mathrm{Cr}_{\mathrm{x}}\right)_{2} \mathrm{Fe}_{36} \mathrm{O}_{60}\right) \text { for different values of } x\end{array}$ & $8.2-12.4$ \\
\hline 27 & $\begin{array}{l}\text { U-type hexaferrite } \\
\left.\mathrm{Ba}_{4}\left(\mathrm{Co}_{1-3 \mathrm{x}} \mathrm{Cr}_{\mathrm{x}}\right)_{2} \mathrm{Fe}_{36} \mathrm{O}_{60}\right) \text { for different values of } x\end{array}$ & $8.2-12.4$ \\
\hline 28 & $\begin{array}{l}\text { U-type hexaferrite } \\
\left.\mathrm{Ba}_{4}\left(\mathrm{Co}_{1-3 \mathrm{x}} \mathrm{Cr}_{\mathrm{x}}\right)_{2} \mathrm{Fe}_{36} \mathrm{O}_{60}\right) \text { for different values of } x\end{array}$ & $8.2-12.4$ \\
\hline \multicolumn{3}{|c|}{$\begin{array}{l}\text { Polyaniline (PA)/expanded graphite (EG) composites mixed at different weight percentages (wt. \%) with novolac phenolic resin } \\
\text { (NPR) (PA/EG }- \text { NPR) }\end{array}$} \\
\hline 29 & $x=0.15, \quad 10$ wt. $\%$ & \\
\hline 30 & 20 wt. $\%$ & \\
\hline 31 & 30 wt. $\%$ & \\
\hline 32 & 10 wt. $\%$ & \\
\hline 33 & 20 wt. $\%$ & $8.2-12.4$ \\
\hline 34 & 30 wt. $\%$ & \\
\hline 35 & 10 wt. $\%$ & \\
\hline 36 & 20 wt. $\%$ & \\
\hline 37 & $x=0.00, \quad 30 w t . \%$ & \\
\hline
\end{tabular}

Table 5. The layer properties and reflection coefficients of Des4, Des5, Des6 and Des7

\begin{tabular}{|c|c|c|c|c|c|c|}
\hline \multirow[t]{2}{*}{ L.\# } & \multicolumn{3}{|c|}{$\begin{array}{c}\text { Des } 4 \\
2-8 \mathrm{GHz} \quad 0^{0}-60^{0} \mathrm{TE} / \mathrm{TM} \text { pol. }\end{array}$} & \multicolumn{3}{|c|}{$\begin{array}{c}\text { Des5 } \\
\text { 8.2-12.4 GHz } 0^{0}-60^{0} \mathrm{TE} / \mathrm{TM}\end{array}$} \\
\hline & Mat. \# & \multicolumn{2}{|c|}{ Thick. (mm) } & Mat \# & \multicolumn{2}{|c|}{ Thick. (mm) } \\
\hline 1 & 19 & \multicolumn{2}{|c|}{3.975} & 17 & \multicolumn{2}{|c|}{0.095} \\
\hline 2 & 20 & \multicolumn{2}{|c|}{1.072} & 36 & \multicolumn{2}{|c|}{2.551} \\
\hline 3 & 24 & \multicolumn{2}{|c|}{1.954} & 37 & \multicolumn{2}{|c|}{0.894} \\
\hline 4 & 18 & \multicolumn{2}{|c|}{1.144} & 17 & \multicolumn{2}{|c|}{0.517} \\
\hline .ే & \multicolumn{3}{|c|}{ Angle of incidence } & \multicolumn{3}{|c|}{ Angle of incidence } \\
\hline 苾 & $0^{0}$ & $-12.05 \mathrm{TE}$ & $-12.05 \mathrm{Tм}$ & $0^{0}$ & $-11.96 \mathrm{TE}$ & $-11.96 \mathrm{TM}$ \\
\hline$\stackrel{0}{\frac{\pi}{0}}$ & $10^{\circ}$ & $-12.02 \mathrm{TE}$ & $-12.23 \mathrm{TM}$ & $10^{0}$ & $-12.01 \mathrm{TE}$ & $-12.16 \mathrm{TE}$ \\
\hline \multirow{5}{*}{ 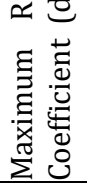 } & $20^{\circ}$ & $-11.82 \mathrm{TE}$ & $-12.76^{\mathrm{TM}}$ & $20^{\circ}$ & $-12.11 \mathrm{TE}$ & $-12.74 \mathrm{TE}$ \\
\hline & $30^{\circ}$ & $-11.22 \mathrm{TE}$ & $-13.72 \mathrm{TM}$ & $30^{\circ}$ & $-12.19 \mathrm{TE}$ & $-13.62 \mathrm{TE}$ \\
\hline & $40^{\circ}$ & $-9.96 \mathrm{TE}$ & $-15.16 \mathrm{TM}$ & $40^{\circ}$ & $-12.05 \mathrm{TE}$ & $-14.42 \mathrm{TE}$ \\
\hline & $50^{\circ}$ & $-8.26 \mathrm{TE}$ & $-16.30 \mathrm{TM}$ & $50^{\circ}$ & $-11.24 \mathrm{TE}$ & $-14.11 \mathrm{TE}$ \\
\hline & $60^{\circ}$ & $-6.29 \mathrm{TE}$ & $-11.79 \mathrm{TM}$ & $60^{\circ}$ & $-8.81 \mathrm{TE}$ & $-11.40 \mathrm{TE}$ \\
\hline TT & \multicolumn{3}{|c|}{$8.14(\mathrm{~mm})$} & \multicolumn{3}{|c|}{$4.05(\mathrm{~mm})$} \\
\hline NL & \multicolumn{3}{|c|}{4} & \multicolumn{3}{|c|}{4} \\
\hline \multirow{3}{*}{ L.\# } & \multirow{3}{*}{\multicolumn{3}{|c|}{$\begin{array}{l}\text { Des6 } \\
\text { 12-18 GHz } 0^{0}-60^{0} \mathrm{TE} / \mathrm{TM} \text { pol. } \\
\text { Mat. \# }\end{array}$}} & \multirow{2}{*}{\multicolumn{3}{|c|}{$\begin{array}{l}\text { Des7 } \\
0^{0}-60^{\circ}\end{array}$}} \\
\hline & & & & & & \\
\hline & & & & Mat \# & & \\
\hline 1 & 19 & \multicolumn{2}{|c|}{1.851} & 19 & \multicolumn{2}{|c|}{2.980} \\
\hline 2 & 20 & \multicolumn{2}{|c|}{0.803} & 21 & \multicolumn{2}{|c|}{1.384} \\
\hline 3 & 17 & \multicolumn{2}{|c|}{0.298} & 22 & \multicolumn{2}{|c|}{0.881} \\
\hline 4 & 22 & & & 18 & & \\
\hline 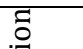 & & gle of incid & & & gle of incic & \\
\hline & $0^{0}$ & $-9.99 \mathrm{TE}$ & $-9.99 \mathrm{TM}$ & $0^{0}$ & $-8.33^{\mathrm{TE}}$ & $-8.33 \mathrm{TM}$ \\
\hline$\stackrel{0}{\mathscr{0}} \cong$ & $10^{\circ}$ & $-9.97 \mathrm{TE}$ & $-10.16^{\mathrm{TM}}$ & $10^{0}$ & $-8.26^{\mathrm{TE}}$ & $-8.48 \mathrm{TM}$ \\
\hline$\simeq$ E & $20^{\circ}$ & $-9.89 \mathrm{TE}$ & $-10.70 \mathrm{TM}$ & $20^{\circ}$ & $-8.05^{\mathrm{TE}}$ & $-8.97 \mathrm{TM}$ \\
\hline 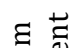 & $30^{\circ}$ & $-9.70 \mathrm{TE}$ & $-11.71 \mathrm{TM}$ & $30^{\circ}$ & $-7.68^{\mathrm{TE}}$ & -9.89 тм \\
\hline 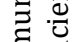 & $40^{\circ}$ & $-9.27 \mathrm{TE}$ & -13.35 тм & $40^{\circ}$ & $-7.11^{\mathrm{TE}}$ & -11.47 тм \\
\hline 気 㫣 & $50^{\circ}$ & $-8.44 \mathrm{TE}$ & $-15.59 \mathrm{TM}$ & $50^{\circ}$ & $-6.28^{\mathrm{TE}}$ & $-11.16^{\mathrm{TM}}$ \\
\hline$\sum^{\pi} \stackrel{0}{0}$ & $60^{\circ}$ & $-7.04 \mathrm{TE}$ & -15.42 тм & $60^{\circ}$ & $-5.02^{\mathrm{TE}}$ & -9.45 тм \\
\hline TT & & $3.24(\mathrm{~mm}$ & & & $6.056(\mathrm{mr}$ & \\
\hline NL & & 4 & & & 4 & \\
\hline
\end{tabular}


Table 6. The results selected from 50 pareto points obtained by TO-ABC algorithm for Des4 (2-8 GHz)

\begin{tabular}{lllllllll}
\hline & Layer sequence & $\mathrm{d} 1$ & $\mathrm{~d} 2$ & $\mathrm{~d} 3$ & $\mathrm{~d} 4$ & $\mathrm{RTE}$ & $\mathrm{RTM}$ & $\mathrm{TT}$ \\
\hline 28 & {$[19-21-20-18]$} & 3.535 & 0.780 & 0.299 & 1.232 & -5.051 & -10.600 & 5.845 \\
29 & {$[19-21-20-18]$} & 3.118 & 1.481 & 0.458 & 1.082 & -4.928 & -10.794 & 6.138 \\
30 & {$[19-21-20-18]$} & 4.000 & 0.770 & 0.012 & 1.422 & -5.171 & -11.162 & 6.204 \\
31 & {$[19-20-18]$} & 4.000 & 1.082 & 1.461 & & -5.238 & -11.749 & 6.543 \\
32 & {$[19-20-18]$} & 4.000 & 1.159 & 1.422 & & -5.268 & -11.827 & 6.581 \\
33 & {$[19-20-24-18]$} & 3.975 & 1.072 & 1.954 & 1.144 & -6.290 & -11.790 & 8.140 \\
34 & {$[19-24-17]$} & 4.000 & 3.802 & 1.508 & & -6.048 & -12.813 & 9.310 \\
\hline
\end{tabular}

Table 7. The results selected from 38 pareto points obtained by TO-ABC algorithm for Des5 (8.2-12.4 GHz)

\begin{tabular}{ccccccccc}
\hline & Layer sequence & $\mathrm{d} 1$ & $\mathrm{~d} 2$ & $\mathrm{~d} 3$ & $\mathrm{~d} 4$ & $\mathrm{RTE}$ & $\mathrm{RTM}$ & $\mathrm{TT}$ \\
\hline 18 & {$[25-29-30-25]$} & 0.684 & 0.828 & 0.074 & 0.757 & -6.466 & -12.809 & 2.343 \\
19 & {$[18-35-26-18]$} & 0.095 & 2.542 & 0.478 & 0.396 & -6.884 & -14.660 & 3.512 \\
20 & {$[17-36-37-17]$} & 0.095 & 2.551 & 0.894 & 0.517 & -8.812 & -11.405 & 4.058 \\
21 & {$[36-36-17-24]$} & 3.358 & 0.862 & 0.655 & 0.380 & -8.040 & -14.950 & 5.254 \\
22 & {$[32-34-20-23]$} & 3.336 & 0.443 & 1.195 & 0.538 & -7.398 & -15.295 & 5.513 \\
23 & {$[29-23-34]$} & 4.000 & 0.622 & 1.731 & & -7.802 & -16.404 & 6.353 \\
24 & {$[30-29-26-27]$} & 1.442 & 3.026 & 2.032 & 2.628 & -9.413 & -12.639 & 9.129 \\
\hline
\end{tabular}

Table 8. The results selected from 40 pareto points obtained by TO-ABC algorithm for Des6 $(12-18 \mathrm{GHz})$

\begin{tabular}{lllllllll} 
& Layer sequence & $\mathrm{d} 1$ & $\mathrm{~d} 2$ & $\mathrm{~d} 3$ & $\mathrm{~d} 4$ & $\mathrm{RTE}$ & $\mathrm{RTM}$ & $\mathrm{TT}$ \\
\hline 9 & {$[19-20-17-22]$} & 1.061 & 1.006 & 0.297 & 0.185 & -5.379 & -12.993 & 2.549 \\
10 & {$[19-20-17-22]$} & 1.279 & 0.837 & 0.243 & 0.289 & -5.547 & -13.839 & 2.648 \\
11 & {$[19-20-17-22]$} & 1.362 & 0.791 & 0.250 & 0.312 & -5.743 & -14.561 & 2.715 \\
12 & {$[19-20-17-22]$} & 1.486 & 0.761 & 0.298 & 0.283 & -5.793 & -14.516 & 2.827 \\
13 & {$[19-20-17-22]$} & 1.461 & 0.801 & 0.298 & 0.291 & -5.846 & -14.192 & 2.851 \\
14 & {$[19-20-17-22]$} & 1.560 & 0.814 & 0.298 & 0.291 & -6.199 & -12.922 & 2.963 \\
15 & {$[19-20-17-22]$} & 1.851 & 0.803 & 0.298 & 0.291 & -7.036 & -9.994 & 3.243 \\
\hline
\end{tabular}

Table 9. The results selected from 19 pareto points obtained by TO-ABC algorithm for Des7 (2-18 GHz)

\begin{tabular}{lllllllll}
\hline & Layer sequence & $\mathrm{d} 1$ & $\mathrm{~d} 2$ & $\mathrm{~d} 3$ & $\mathrm{~d} 4$ & $\mathrm{RTE}$ & $\mathrm{RTM}$ & $\mathrm{TT}$ \\
\hline 11 & {$[19-24-21-18]$} & 2.894 & 0.093 & 1.925 & 0.837 & -4.960 & -8.763 & 5.749 \\
12 & {$[19-21-22-18]$} & 2.781 & 1.387 & 0.742 & 0.866 & -4.811 & -9.647 & 5.775 \\
13 & {$[19-24-21-18]$} & 2.894 & 0.232 & 1.866 & 0.849 & -4.897 & -8.960 & 5.842 \\
14 & {$[19-24-21-18]$} & 2.894 & 0.353 & 1.783 & 0.897 & -4.872 & -9.152 & 5.928 \\
15 & {$[19-21-22-18]$} & 2.980 & 1.384 & 0.881 & 0.811 & -5.026 & -8.330 & 6.056 \\
16 & {$[19-21-22-18]$} & 2.885 & 1.598 & 0.898 & 0.771 & -5.000 & -9.247 & 6.153 \\
17 & {$[19-24-20-18]$} & 2.796 & 1.334 & 1.526 & 1.098 & -4.911 & -9.158 & 6.754 \\
\hline
\end{tabular}

As listed in the Table 5, both RTE and RTM of Des7 are below $-7 \mathrm{~dB}$ for the all angles of incidence between $0^{0}$ $40^{\circ}$. Although the all designs in this section are optimized using limited number materials, the obtained satisfactory results clearly demonstrate the superiority of the TO-ABC method.

As shown in Table 5, reflection coefficients of the Des6 is below $-9.27 \mathrm{~dB}$ for entire frequency band and angles of incident between $0^{\circ}-40^{\circ}$. Since the Des7 is optimized to operate at broad-band $(2-18 \mathrm{GHz})$, as shown in Table 9, the maximum reflection coefficient is found about $-5 \mathrm{~dB}$ for whole angles of incidence.

\section{CONCLUSION}

In the last 25 years, optimization based many studies have been carried out for MRA design. While the majority of these studies were performed with singleobjective optimization, some of them were performed with double-objective approaches. However, in order to fully optimize an MRA structure, RTE, RTM and TT must be simultaneously optimized. Thanks to the proposed TO-ABC algorithm, RTE, RTM and TT are simultaneously optimized for the first time and the ES of the objective functions are successfully obtained. Three MRA structures designed with virtual materials are compared with the latest MRAs in the literature and the performance of the proposed method is demonstrated. In addition, thanks to the developed GUI, a practical and easy user interface for MRA design is also provided.

Furthermore, four different broad-band wide-angle MRAs operating in the frequency ranges of $2-18 \mathrm{GHz}$ are designed using 21 real-materials presented in recent years. Thanks to developed TO-ABC algorithm, despite the limited number of materials, all possible solutions providing the specified parameters are achieved and successful MRA structures are designed. With the help of proposed GUI, MRA designs can be practically realized without the need for complex computer scripts. Since the results obtained in this study provide a new perspective 
for MRA design, triple-objective approach can be developed for different optimization techniques in further studies.

\section{REFERENCES}

Asi M \& Dib N I (2010). Design of multilayer microwave broadband absorbers using central force optimization. Progress in Electromagnetic Research, 26, 101-113.

Deb K, Pratap A, Agarwal S \& Meyarivan T (2002). A fast and elitist multiobjective genetic algorithm: NSGA-II. IEEE Trans. Evol. Comput. 6, 182-197. https://doi.org/10.1109/4235.996017

Goudos S K (2009). Design of microwave broadband absorbers using a self-adaptive differential evolution algorithm. International Journal of RF and Microwave Computer-Aided Engineering, 19(3), 364-372.

Goudos S K \& Sahalos J N (2006a). Design of Broadband Radar Absorbing Materials using Particle Swarm Optimization, Proceedings of EMC Europe, 11111116.

Goudos S K \& Sahalos J N (2006b). Microwave absorber optimal design using multi-objective particle swarm optimization. Microwave and Optical Technology Letters, 48, 1553-1558.

Huo Y, Zhuang Y, Gu J \& Ni S (2015). Elite-guided multiobjective artificial bee colony algorithm. Applied Soft Computing 32, 199-210. https://doi.org/https://doi.org/10.1016/j.asoc.201 5.03.040

Jiang L, Cui J, Shi L \& Li X (2009). Pareto optimal design of multilayer microwave absorbers for wide-angle incidence using genetic algorithms. IET microwaves, antennas \& Propagation, 3(4), 572-579.

Karaboga D \& Basturk B (2007). A powerful and efficient algorithm for numerical function optimization: artificial bee colony (ABC) algorithm. Journal of Global Optimization, 39(3), 459-471.

Kern D J \& Werner D H (2003). A genetic algorithm approach to the design of ultra-thin electromagnetic bandgap absorbers. Microwave and Optical Technology Letters, 38, 61-64. https://doi.org/10.1002/mop.10971

Michielssen E, Sajer J-M, Ranjithan S \& Mittra R (1993). Design of lightweight, broad-band microwave absorbers using genetic algorithms. IEEE
Transactions on Microwave Theory and Technology, 41(6), 1024-1031.

Ranjan P, Choubey A \& Mahto S K (2018). A novel approach for optimal design of multilayer wideband microwave absorber using wind driven optimization technique. AEU-International Journal of Electronics and Communications, 83, 81-87.

Roy S, Mahanti A, Roy S D \& Mahanti G K (2016). Comparison of Evolutionary Algorithms for Optimal Design of Broadband Multilayer Microwave Absorber for Normal and Oblique Incidence. Applied Computational Electromagnics Society Journal, 31(1).

Roy S, Roy S D, Tewary J, Mahanti A \& Mahanti G K (2015). Particle swarm optimization for optimal design of broadband multilayer microwave absorber for wide angle of incidence. Progress in Electromagnics Research, 62, 121-135.

Toktas A, Ustun D \& Tekbas M (2019). Multi-Objective Design of Multi-Layer Radar Absorber Using Surrogate-Based Optimization. IEEE Transations on Microwave Theory and Technology, 67, 3318-3329. https://doi.org/10.1109/TMTT.2019.2922600

Toktas A, Ustun D, Yigit E, Sabanci K \& Tekbas M (2018). Optimally Synthesizing Multilayer Radar Absorbing Material (RAM) Using Artificial Bee Colony Algorithm. Proceedings of International Seminar/Workshop on Direct and Inverse Problems of Electromagnetic and Acoustic Wave Theory, DIPED. IEEE, 237-241. https://doi.org/10.1109/DIPED.2018.8543261

Weile D S, Michielssen E \& Goldberg D E (1996). Genetic algorithm design of Pareto optimal broadband microwave absorbers. IEEE Transactions on Electromagnetic Compatibility, 38(3), 518-525.

Yigit E \& Duysak H (2019)a. Determination of Optimal Layer Sequence and Thickness for Broadband Multilayer Absorber Design Using Double-Stage Artificial Bee Colony Algorithm. IEEE Transactions on Microwave Theory and Techniques, 67(8), 33063317.

Yigit E \& Duysak H (2019)b. electrical_properties.xlsx. IEEE Transactions on Microwave Theory and Technology https://ieeexplore.ieee.org/abstract/document/874 4403/media\#media 